Case Report

\title{
Pulmonary Endarterectomy in a Patient with Immune Thrombocytopenic Purpura
}

\author{
Bedrettin Yıldızeli, ${ }^{1}$ Mehmed Yanartaş, ${ }^{2}$ Sibel Keskin, ${ }^{3}$ Işık Atagündüzz, ${ }^{4}$ and Ece Altınay ${ }^{5}$ \\ ${ }^{1}$ Department of Thoracic Surgery, Marmara University School of Medicine, Istanbul, Turkey \\ ${ }^{2}$ Department of Cardiovascular Surgery, Kartal Koșuyolu Training and Research Hospital, Istanbul, Turkey \\ ${ }^{3}$ Department of Chest Diseases, Muğla Sitkı Koçman University, Muğla, Turkey \\ ${ }^{4}$ Department of Hematology, Marmara University School of Medicine, Istanbul, Turkey \\ ${ }^{5}$ Department of Anaesthesia, Kartal Koşuyolu Training and Research Hospital, Istanbul, Turkey \\ Correspondence should be addressed to Bedrettin Yıldızeli; byildizeli@marmara.edu.tr
}

Received 6 January 2015; Accepted 10 May 2015

Academic Editor: Paolo Vanelli

Copyright ( $\odot 2015$ Bedrettin Ylddizeli et al. This is an open access article distributed under the Creative Commons Attribution License, which permits unrestricted use, distribution, and reproduction in any medium, provided the original work is properly cited.

\begin{abstract}
Immune thrombocytopenic purpura (ITP) patients are at high risk for bleeding complications regarding surgeries involving cardiopulmonary bypass. We report an ITP patient with chronic thromboembolic pulmonary hypertension who underwent uncomplicated pulmonary endarterectomy with receiving postoperative intravenous immunoglobulin (IVIG) therapy. The positive outcome of this case may suggest that pulmonary endarterectomy surgery is performed safely for ITP patients.
\end{abstract}

\section{Introduction}

Immune thrombocytopenic purpura (ITP) is a common hematological disorder which involves immune mediated platelet destruction and impaired platelet production [1]. It is characterized by isolated thrombocytopenia in the absence of other causes or disorders that may be associated with thrombocytopenia [1]. The association of ITP and chronic thromboembolic pulmonary hypertension $(\mathrm{CTEPH})$ represents a complex therapeutic challenge. To our knowledge, no case was reported to describe management of patients with ITP undergoing pulmonary endarterectomy (PEA). We describe our experience of perioperative management in a patient of ITP who underwent surgery.

\section{Case Report}

A 69-year-old female with shortness of breath (New York Heart Association Class IV) and CTEPH for a 1-year period was referred for PEA. Her medical history revealed that she had hypertension, regulated with oral antihypertensive drugs, and type II diabetes mellitus. Routine hematological examination was normal with a platelet count of $228,000 / \mathrm{mm}^{3}$. Her hematologic investigations showed a provisional diagnosis of ITP for 12 years with history of splenectomy 10 years ago. The patient was on chronic corticosteroid and azathioprine therapy on admission.

Her echocardiogram revealed a dilated right ventricle with reduced systolic function and moderate tricuspid regurgitation, but normal left ventricular function with an ejection fraction of $65 \%$. A right heart catheterization revealed slightly elevated pulmonary artery $(\mathrm{PA})$ pressure of $45 / 16 \mathrm{~mm} \mathrm{Hg}$ (mean, $28 \mathrm{~mm} \mathrm{Hg}$ ) and cardiac output (CO) of $3.3 \mathrm{~L} / \mathrm{min}$. Calculated pulmonary vascular resistance (PVR) was 436 dynes $/ \mathrm{s} / \mathrm{cm}^{-5}$. Spiral computed tomographic scanning of her chest showed right pleural effusion and web-like filling defects and endoluminal thromboemboli in her pulmonary vasculature on the right and left lower lobe sides consistent with chronic thromboembolic disease (Figure 1). Ventilation perfusion scanning confirmed the diagnosis of CTEPH and the patient was scheduled for pulmonary endarterectomy. The PEA surgery was performed 


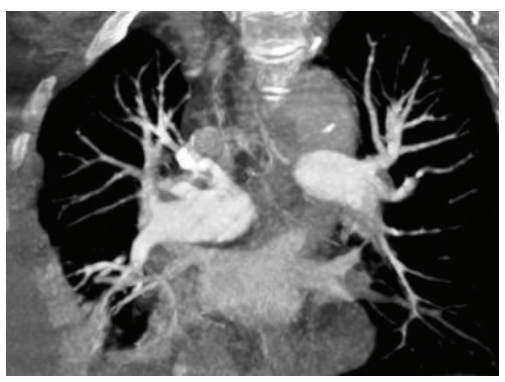

FIgURE 1: Computed tomogram shows right pleural effusion, weblike filling defects, and endoluminal thromboemboli extending into the right and left pulmonary artery.

as previously described [2]. In brief, surgery was performed under general anaesthesia through a median sternotomy and using extracorporeal circulation. After anticoagulation with heparin, activated clotting time (ACT) of 480 seconds was attained and cardiopulmonary bypass $(\mathrm{CPB})$ was initiated. The endarterectomy specimen was circumferentially followed down to the segmental and subsegmental branches of the pulmonary artery in each lobe, until a complete endarterectomy of the pulmonary vascular bed was achieved with periods of circulatory arrest under deep hypothermia $\left(20^{\circ} \mathrm{C}\right)$ for the right and left pulmonary arteries (Figure 2). Patient weaned from CPB without any difficulty and her PVR was decreased to 250 dynes $/ \mathrm{s} / \mathrm{cm}^{-5}$. Bleeding was checked thoroughly and heparin was reversed with protamine to normalize ACT. The chest was closed and the patient was transferred to the intensive care unit and put on ventilator with stable hemodynamics. Six hours later, platelets counts were $158,000 / \mathrm{mm}^{3}$. On the first postoperative day (pod), the patient was put on oral azathioprine $50 \mathrm{mg}$ and oral prednisolone $16 \mathrm{mg}$ daily and subcutaneous injections (s.c.) of low-molecular-weight heparin (LMWH) 0.6 cc twice daily. The platelets counts were $241,000 / \mathrm{mm}^{3}$. On the 2 nd pod platelet counts were decreased to $57,500 / \mathrm{mm}^{3}$, and, instead of LMWH, an indirect factor Xa inhibitor fondaparinux was initiated $2.5 \mathrm{mg} /$ day s.c. and dose of prednisolone was increased to $100 \mathrm{mg}$ daily. After three days of treatment, a low platelet count of $57,000 / \mathrm{mm}^{3}$ was observed. Steroid therapy was stopped and intravenous immunoglobulin (IVIG) was started at a dose of $400 \mathrm{mg} / \mathrm{kg} /$ day for 5 days. Patient was extubated on 5th pod with stable hemodynamics and blood gases. In the meantime the platelet concentrates were transfused between first and 4th pod. Platelet counts were assessed daily and showed a rising trend. On 7th pod, the platelets counts were $183,000 / \mathrm{mm}^{3}$. And she was transferred to the ward. IVIG was switched to oral prednisolone treatment, and also azathioprine, fondaparinux, and warfarin therapy was continued. The patient was discharged on 14th pod with a platelet count of $125,000 / \mathrm{mm}^{3}$ and with substantial functional and hemodynamic improvement and with advice to continue her medications azathioprine $50 \mathrm{mg}$ and prednisolone $16 \mathrm{mg}$ daily and regularly follow up with a hematologist.

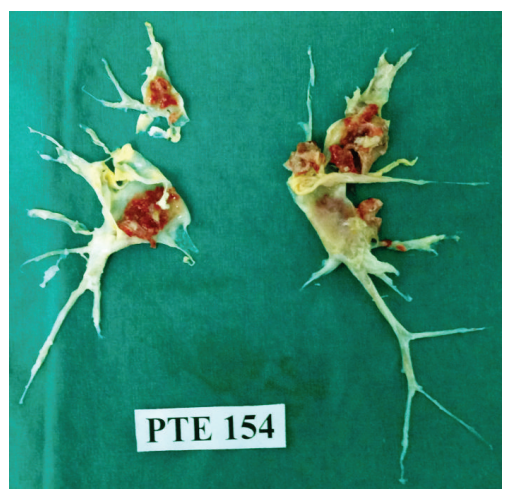

FIGURE 2: Photograph shows the specimen from our patient's pulmonary endarterectomy.

\section{Discussion}

Immune thrombocytopenic purpura is characterized by an abnormally low platelet count of unknown cause. IgG antiplatelet autoantibodies are produced against the platelet glycoprotein IIb/IIIa or GPIb/IX in about $75 \%$ of patients causing both platelet destruction and inhibition of thrombopoiesis [1]. The main problem in patients of ITP is an increased risk of bleeding although bleeding symptoms may not always be present. Concomitantly, the ITP patients also present an increased risk of thrombosis related to the presence of hemostatic factors and chronic steroid therapy.

On the other hand, several investigators have recently found in control retrospective and prospective cohort studies that splenectomy is a risk factor of CTEPH [3]. Splenectomy is associated with venous thrombosis in general and, in particular, with nonresolving and recurrent thrombosis and deep vein thrombosis. One late consequence of nonresolution of venous and pulmonary thromboemboli is CTEPH.

Pulmonary endarterectomy is only curative therapy of CTEPH [4]. The technical details of PEA surgery are well established. By using extracorporeal circulation and circulatory arrest under deep hypothermia $\left(20^{\circ} \mathrm{C}\right)$, endarterectomy specimen is circumferentially followed down to the segmental and subsegmental branches of the pulmonary artery. Although there is no contraindication for PEA surgery, operability of the CTEPH patient should be determined by an experienced PEA team [4].

Bleeding after $\mathrm{CPB}$ surgery is common with about $7 \%$ of patients requiring reoperation to control bleeding [5]. Preoperative thrombocytopenia, CPB, deep hypothermia, and induced thrombocytopenia as well as platelet dysfunction and postoperative anticoagulation are expected to increase risk of pericardial effusion and cardiac tamponade. Chowdhry et al. [5] reported a patient with ITP with severe coronary artery disease and mitral regurgitation who underwent $\mathrm{CABG}$ and mitral valve replacement. Although early outcome of the surgery was uncomplicated, the patient was dead three weeks later with a diagnosis of pericardial tamponade. 
The main treatment of ITP consists of corticosteroids and IVIG. Second and third line therapies, including rituximab, splenectomy, thrombin receptor agonists (TPO-A), and immune-suppressants, are often successful and may cause a long-term increase in the platelet counts [5]. Patients with asymptomatic mild or moderate thrombocytopenia can be followed up with no treatment as platelet counts greater than $5 \times 10^{4} / \mathrm{mm}^{3}$ are usually not associated with clinically important bleeding. Although platelet transfusion may be short acting, it is useful in instances of severe hemorrhage. Infusion of IVIG has an immediate effect in increasing platelet counts as it blocks the crystallizable fragment $(\mathrm{Fc})$ receptors of macrophages, thus avoiding destruction by phagocytosis.

Jubelirer et al. [6] reported that patients with ITP presenting with mild or moderate thrombocytopenia can be successfully supported to control bleeding during or after coronary artery bypass grafting (CABG) with IVIG and/or platelet transfusions.

The present patient had a diagnosis of ITP and CTEPH disease and underwent successful PEA. To our knowledge, no case of PEA for a patient with a history of ITP has previously been reported. She had an uneventful intraoperative course and there was no difficulty in obtaining surgical hemostasis. In the postoperative period also the patient did not have much bleeding and bleeding related complications. The decrease in the platelet count was managed successfully with platelet transfusion, steroids, and IVIG. Patient was discharged with acceptable platelet counts and with advice about taking anticoagulants, timely INR monitoring, and regular follow-up.

We conclude that PEA surgery can be performed safely to a patient with CTEPH associated ITP. We would recommend that, with an accurate and precise postoperative management, ITP is not contraindicated for PEA surgery.

\section{Conflict of Interests}

The authors declare that there is no conflict of interests regarding the publication of this paper.

\section{References}

[1] T. Kühne, "Update on the intercontinental cooperative ITP study group (ICIS) and on the pediatric and adult registry on chronic ITP (PARC ITP)," Pediatric Blood and Cancer, vol. 60, supplement 1, pp. S15-S18, 2013.

[2] B. Yıldızeli, S. Taş, M. Yanartaş et al., "Pulmonary endarterectomy for chronic thrombo-embolic pulmonary hypertension: an institutional experience," European Journal Cardio-Thoracic Surgery, vol. 44, no. 3, pp. e219-e227, 2013.

[3] N. H. Kim and I. M. Lang, "Risk factors for chronic thromboembolic pulmonary hypertension," European Respiratory Review, vol. 21, no. 123, pp. 27-31, 2012, Review.

[4] N. H. Kim, M. Delcroix, D. P. Jenkins et al., "Chronic thromboembolic pulmonary hypertension," Journal of the American College of Cardiology, vol. 62, no. 25, supplement, pp. D92-D99, 2013.

[5] V. Chowdhry, B. B. Mohanty, and D. Probodh, "Cardiac surgery in a patient with immunological thrombocytopenic purpura: complications and precautions," Annals of Cardiac Anaesthesia, vol. 16, no. 2, pp. 147-150, 2013.

[6] S. J. Jubelirer, L. Mousa, U. Reddy, M. Mir, and C. A. Welch, "Coronary artery bypass grafting (CABG) in patients with immune thrombocytopenia (ITP): a community hospital experience and review of the literature," The West Virginia Medical Journal, vol. 107, no. 6, pp. 10-14, 2011. 


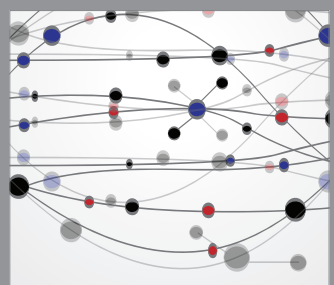

The Scientific World Journal
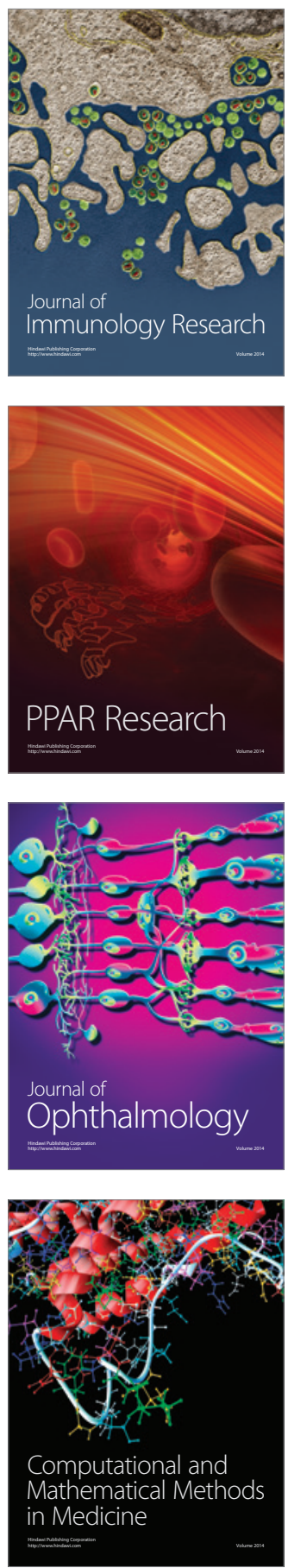

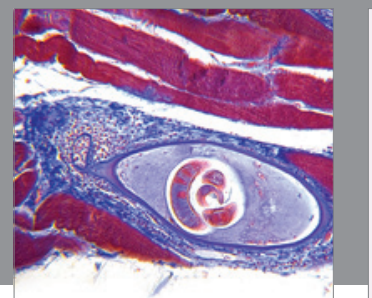

Gastroenterology

Research and Practice
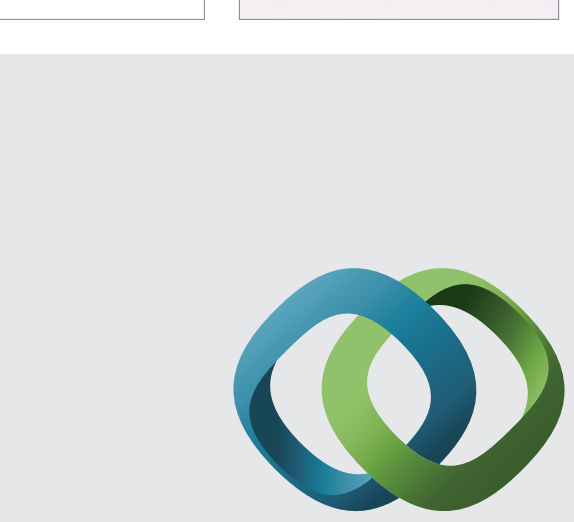

\section{Hindawi}

Submit your manuscripts at

http://www.hindawi.com
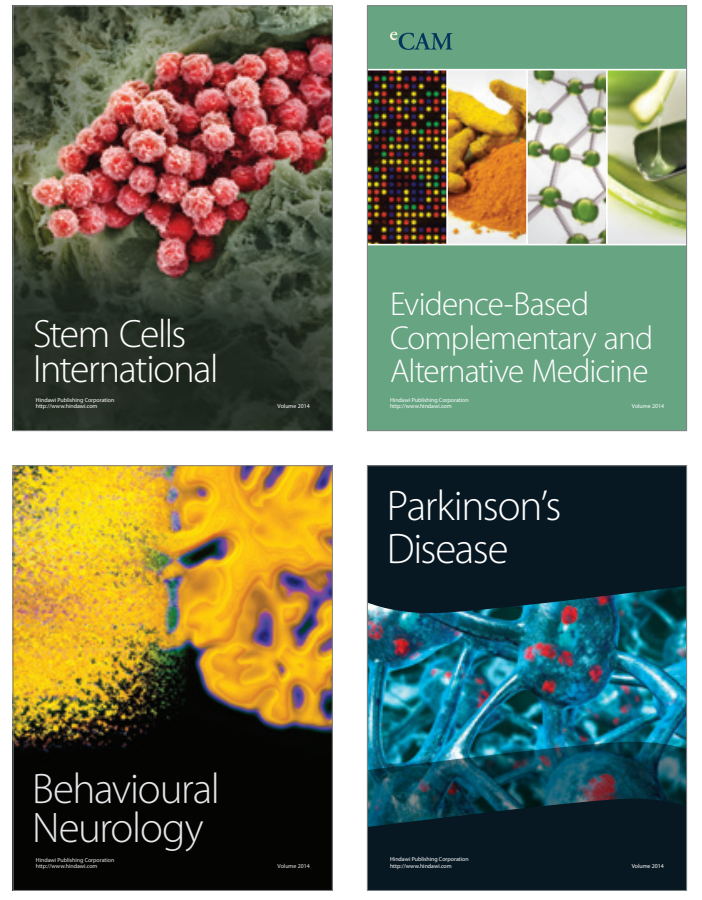
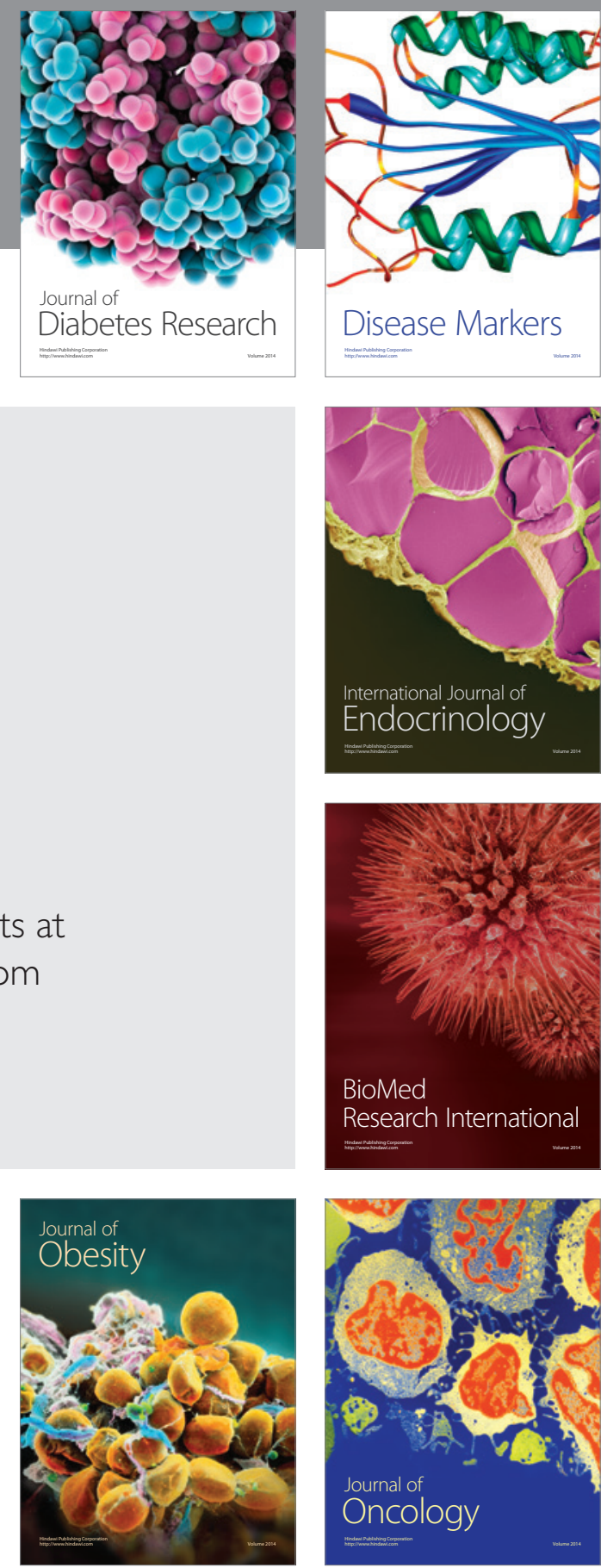

Disease Markers
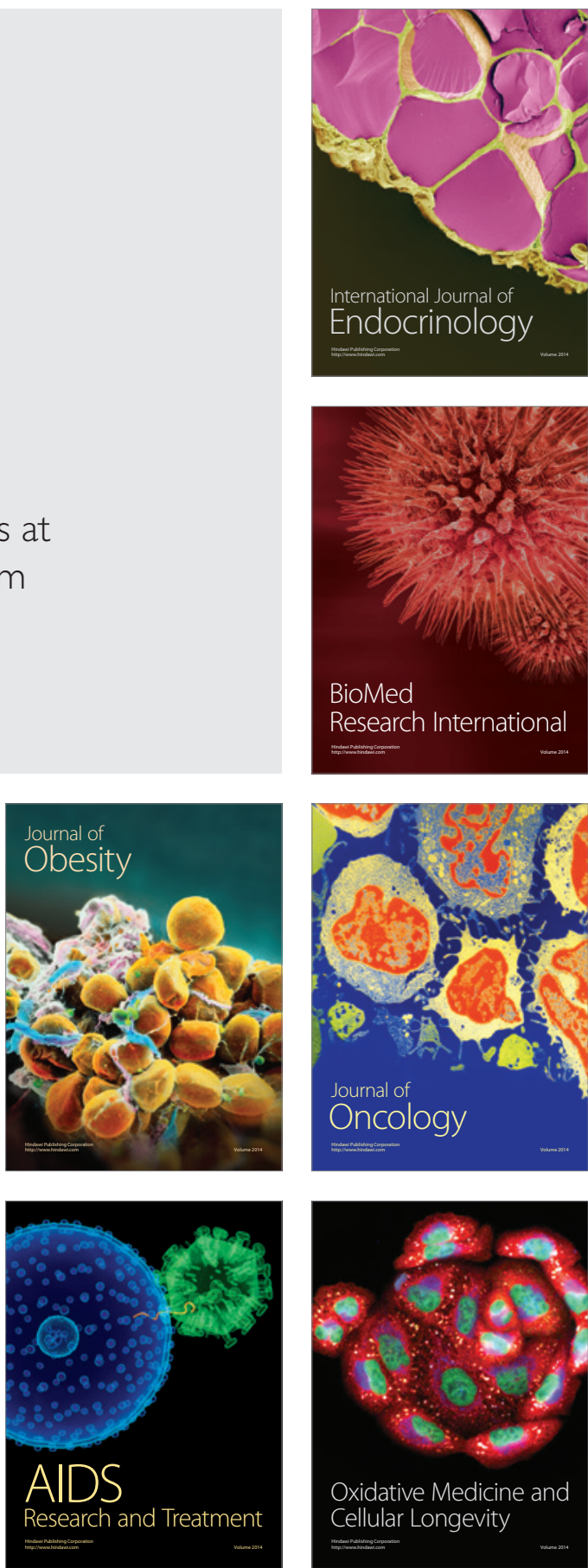